\title{
The Lyot Project: Understanding the AEOS Adaptive Optics PSF
}

\author{
Russell B. Makidon ${ }^{1,5}$, A. Sivaramakrishnan ${ }^{2,5}$, R. Soummer ${ }^{2,5}$, \\ B. R. Oppenheimer ${ }^{2}$, L. C. Roberts, Jr. ${ }^{3}$, J. R. Graham ${ }^{4,5}$, \\ and M. D. Perrin ${ }^{4,5}$ \\ ${ }^{1}$ Space Telescope Science Institute, 3700 San Martin Drive, MD 21218, USA \\ ${ }^{2}$ Department of Astrophysics, American Museum of Natural History, \\ 79th Street at Central Park West, New York NY 10024, USA \\ ${ }^{3}$ The Boeing Company, 535 Lipoa Pkwy, Suite 200, Kihei, HI 96753, USA \\ ${ }^{4}$ Astronomy Department, University of California, Berkelely CA 94000, USA \\ ${ }^{5}$ NSF Center for Adaptive Optics
}

\begin{abstract}
Adaptive optics ( $\mathrm{AO})$ systems have significantly improved astronomical imaging capabilities over the last decade, and are revolutionizing the kinds of science possible with 4$5 \mathrm{~m}$ class ground-based telescopes. A thorough understanding of AO system performance at the telescope can enable new frontiers of science as observations push AO systems to their performance limits. We look at the understanding we have gained from recent Lyot Project images at the Advanced Electro-Optical System (AEOS) $3.6 \mathrm{~m}$ telescope to show how progress made in improving WFR can be measured directly in improved science images. We describe how wave front errors affect the AO point-spread function (PSF), and model details of AEOS AO to simulate a PSF which matches the actual AO PSF in the astronomical H-band. Finally, we estimate the impact of improvements to wave front reconstruction techniques on diffractionlimited coronagraphy with the Lyot Project near-infrared coronagraph.
\end{abstract}

Keywords. instrumentation: adaptive optics, planetary systems: protoplanetary disks.

\section{Introduction}

The $3.6 \mathrm{~m}$ AEOS telescope, part of the U.S. Air Force Research Laboratory's Maui Space Surveillance System (MSSS), is arguably the best telescope on which to develop the nascent field of Extreme Adaptive Optics (ExAO). This is a result both of the telescope's location at a prime astronomical site possessing good seeing and, more importantly, the AEOS adaptive optics system's 941-actuator deformable mirror, with 35 actuators spanning the diameter of the DM. This provides the highest actuator density available to any civilian astronomical observing program, with a projected actuator spacing of order $0.11 \mathrm{~m}$ per actuator at the primary mirror, and currently produces images with Strehl ratios (SRs) of typically $\sim 0.15$ in the $I$-band for objects brighter than 6 th magnitude and Strehl ratios as high as $\sim 0.25$ observed during upper-quartile seeing conditions (see Roberts \& Neyman (2002), Makidon et al. (2005)).

The Lyot Project near-infrared coronagraph, at coudé focus, delivers $H$-band SRs in the $\sim 0.65-0.83$ range and occasionally higher. This near-IR performance agrees in the main with predictions based on results from simple AO simulations anchored in Palomar and AEOS AO data (see Makidon et al. (2005), Sivaramakrishnan et al. (2001)). However, dynamic range with the coronagraph still falls short of predictions based on numerical modeling.

In order to make best use of the advantages offered by AEOS for the Lyot Project coronagraph, we first needed to understand the characteristics of the AO system. For 
the Lyot Project, we have found that one of the best ways to understand the AEOS AO system - which is a facility system not associated with the Lyot Project coronagraph is through modeling the PSF itself.

\section{Modeling AEOS Adaptive Optics}

A detailed simulation of an $\mathrm{AO}$ system including all aspects of wave front sensing, reconstruction, and correction requires substantial computational effort. For some purposes it is sufficient to model the action of an AO system as a transfer function or spatial frequency filter acting on the incident wave front. Following the method presented in Sivaramakrishnan et al. (2001), we assume we use a high-pass filter with a power law distribution in spatial frequency space to mimic the action of the AEOS AO system,

$$
F(k)= \begin{cases}\left(k / k_{A O}\right)^{n}, & \text { for } k<k_{A O} \\ 1, & \text { otherwise. }\end{cases}
$$

In Makidon et al. (2003), we used a 'parabolic' filter (e.g. $n=2$ ) to model this AO system. However, further refinement of our simulations based on comparision with early AEOS $I$-band imaging data have shown that an $n=0.9$ power law distribution best fits the AEOS system. We discuss this in further detail in Makidon et al. (2005).

We simulate a set of $H$-band images by generating thirty-seven monochromatic images across the bandpass, from $\lambda=1.45$ to $1.81 \mu \mathrm{m}$. We assume a single-layer, Kolmogorovspectrum phase screen to represent atmospheric turbulence, and use the $n=0.9$ power law filter in spatial frequency space to represent correction of the incoming wave front by the AEOS AO system. We assume we are in the Fraunhofer regime throughout our simulations, where the image plane is the Fourier Transform of the pupil plane and vice versa.

Our simulations produced model PSFs which matched many of the features of the AEOS AO direct imaging PSF. However, details of the coronagraphic images suggest other sources of static phase aberration remained uncorrected in the optical train.

\section{The Lyot Project: First Light}

First light with the Lyot Project coronagraph at the AEOS $3.63 \mathrm{~m}$ telescope and AO system was in March 2004 (see Fig. 1). While the coronagraph performed at an extremely high level, as predicted from laboratory tests and simulation, the presence of strong and persistent residual speckles led us to consider elements in the AEOS AO optical train as potential sources of phase aberrations in the PSF.

One of the challenges in analyzing coronagraphic image data from this or any other high-Strehl ratio system is to understand the sources of residual speckles, and how to mitigate the effects of these speckles on coronagraphic imaging. For AEOS, we are working to understand the speckles produced by phase aberrations in the telescope and adaptive optics system upstream of the coronagraph optics, as well as issues related to scintillation and pupil illumination.

\section{Modeling Phase Errors in AEOS Optics}

In an attempt to understand the residual speckle structure observed in coronagraphic images acquired with the AEOS AO system, we considered sources of phase error in the telescope and AO optical train, including pinned actuators on the AO systems deformable mirror (DM). As has been reported by Gullapalli et al. (2000), there are a number of 


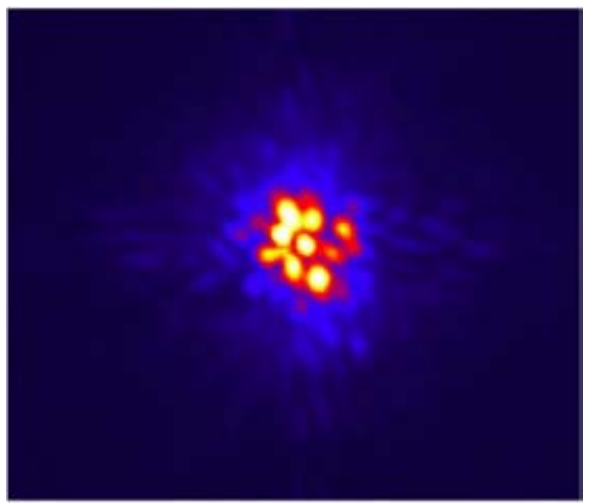

Figure 1. Coronagraphic image of a nearby star observed with the Lyot Project coronagraph at the AEOS $3.63 \mathrm{~m}$ telescope in March 2004. This image was acquired with the Kermit Infrared Camera Perrin et al. (2004) behind the Lyot Projects 0.35" Focal Plane Mask in the $H$-band $(4 \lambda / D$ diameter $)$ and optimized Lyot stop used to suppress light from central star. Residual speckles dominate the noise in this image (see Racine et al. (1999)). Of note is the presence of symmetric and anti-symmetric speckle structure, as predicted from the first- and second-order terms of the PSF expansion of Perrin et al. (2003).
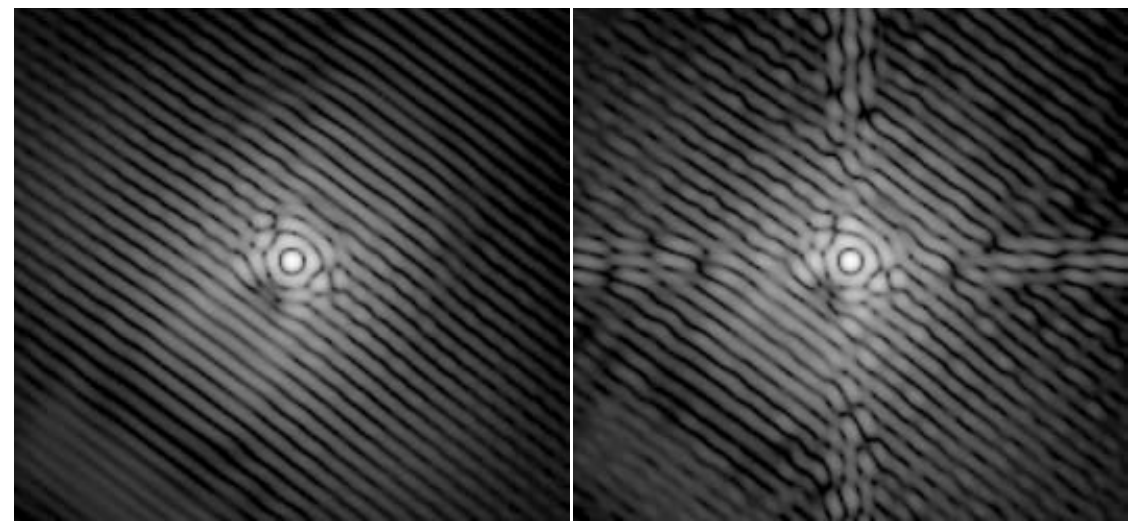

Figure 2. Simulated monochromatic coronagraphic PSFs at $\lambda=1.63 \mu \mathrm{m}$ resolution assuming the Lyot Project coronagraphs $445 \mu \mathrm{m}$-diameter Focal Plane Mask and Lyot Stop. The PSF at left assumes five Gaussian phase aberrations of varied depths in the pupil plane representing pinned DM actuators. Residual speckle structure close to the PSF core dominates the image. The PSF at right includes the same five Gaussian phase aberrations in the pupil plane as well as a secondary mirror support structure that is misaligned with respect to the Lyot Mask. These PSFs are shown at $\lambda / 4 D$ resolution on a logarithmic grayscale, with the upper limit of the grayscale at right $1 / 100$ th that at left. Noise sources due to Poisson statistics or detector effects are absent from these simulations.

actuators on the AEOS DM that are pinned at levels below the nominal zero-level of the DM. Here, we model a DM with five pinned actuators, and represent each pinned actuator as a phase aberration in the form of a Gaussian with FWHM of 1/34th of the pupil diameter, with the a depth of this phase aberration dependent on the physical characteristics of the DM. We then propagate this phase aberration through our simulated AO system and the Lyot Project coronagraph optics. In these simulations, we assume a focal plane mask (FPM) of diameter $445 \mu \mathrm{m}$ in an $\mathrm{f} / 70$ beam followed by an optimallyundersized Lyot stop matched to this FPM (see Figure 2 - left). 
In addition, we considered the situation where, in addition to phase errors induced by the DM, we see phase aberrations due to the incorrect masking the AEOS secondary mirror (SM) support structure in the Lyot pupil (e.g., as in the case of misalignment). We show this for the monochromatic case in Fig. 2 (right). As in the previous example, we see residual speckle close to the PSF core dominating the coronagraphic image. However, the presence of unmasked secondary supports in the Lyot pupil show a significant contribution to the coronagraphic image from a radius of $\sim 12 \lambda / D$ from the PSF core, translating to $1.05^{\prime \prime}$ at the Kermit Infrared Camera with the Lyot Project coronagraph.

\section{Conclusions}

As ground-based and space-based coronagraphic imaging systems push to higher and higher contrasts, the control of - and understanding of - phase aberrations induced by intervening optics becomes more and more important. Knowledge of how those phase aberrations create structure in the final image plane will be necessary for correct interpretation of the observations. Indeed, evern for very high-Strehl imaging systems such as the Advanced Camera for Surveys on the Hubble Space Telescope, it is the presence of residual phase aberrations of the optics which ultimately limits the performance of HST as a high-dynamic range instrument (Krist 2004, private communication).

\section{Acknowledgements}

The authors are grateful to D. T. Gavel, G. Smith, and P. E. Hodge for helpful discussions, and to the the staff of the Maui Space Surveillance System for their assistance in taking the Lyot Project data. R.S. and M.D.P. are supported by NASA Michelson Postdoctoral and Graduate Fellowships, respectively, under contract to the Jet Propulsion Laboratory (JPL) funded by NASA. The JPL is managed for NASA by the California Institute of Technology. The research presented here was supported by the STScI Director's Discretionary Research Fund, NSF grants AST-0088316 and AST-0215793, and AFRL/DE through Contract Number F29601-00-D-0204. Our work has also been supported by the National Science Foundation Science and Technology Center for Adaptive Optics, managed by the University of California at Santa Cruz under cooperative agreement No. AST - 9876783. This research made use of the SIMBAD database, operated at CDS, Strasbourg, France.

\section{References}

Gullapalli, S. N., Abreu, R., Rappoport, W. M., Zmek, W. P., \& Pringle, R. 2000, Proc. SPIE 3931, 285-299

Makidon, R. B., Sivaramakrishnan, A., Roberts, L. C., Oppenheimer, B. R., \& Graham, J. R. Proc. SPIE 4860, 315-323

Makidon, R. B., Sivaramakrishnan, A., Perrin, M. D., Roberts, L. C., Jr., Oppenheimer, B. R., Soummer, R., \& Graham, J. R. 2005, PASP 117, 831

Perrin, M. D., Sivaramakrishnan, A., Makidon, R. B., Oppenheimer, B. R., \& Graham, J. R. 2003, ApJ 596, 702

Perrin, M. D., Graham, J. R., Trumpis, M., Kuhn, J., Whitman, K., Coulter, R., Lloyd, J. P., \& Roberts, L. C., Jr. 2004, 2003 AMOS Tech. Conf.

Racine, R., Walker, G. A. H., Nadeau, D., Doyon, R., \& Marois, C. 1999, PASP 111, 587

Roberts, L. C., Jr. \& Neyman, C. R. 2002, PASP 114, 1280

Sivaramakrishnan, A., Koresko, C. D., Makidon, R. B., Berkefeld, T., \& Kuchner, M. J. 2001, ApJ 552, 397 